\section{On a collection of praying mantids (Insecta: Mantodea) from Goa, India, with new distribution records}

\author{
M.C. Vyjayandi ${ }^{1}$, R.S. Rajeesh ${ }^{2}$, P. Sajin John ${ }^{2}$ \\ \& M.M. Dhanasree ${ }^{2}$ \\ 1,2 Department of Zoology, Providence Women's College, \\ Kozhikode, Kerala 673009, India \\ Email: ${ }^{1}$ vyji@rediffmail.com
}

Mantid fauna of southern India is very rich. Out of the 162 species of mantids under 68 genera reported from India, 45 species under 35 genera are known from southern India (Mukherjee et al. 1995). Only one species was reported from Goa, which is one of the smallest states of India located in the western coast. Serious attempts for field surveys and collection of mantids have ever been carried in the region. The present paper is based on some mantid species from Goa collected during a field survey conducted during the months of SeptemberOctober 2008. A total of six species of mantids under six genera are collected and reported for the first time from Goa, India.

The state of Goa is located within 15048'00"$14^{\circ} 53^{\prime} 54^{\prime \prime} N$ \& $74^{\circ} 20^{\prime} 13^{\prime \prime}-73^{\circ} 40^{\prime} 33^{\prime \prime} E$ and bounded on the north by Sindhudurg District of Maharashtra State, on the west by the Arabian Sea, on the south by Karwar District of Karnataka State and on the east by Belgaum District of Karnataka State. The climate of Goa is warm and humid for most of the year and the month of May is the hottest,

Date of publication (online): 26 November 2010

Date of publication (print): 26 November 2010

ISSN 0974-7907 (online) | 0974-7893 (print)

Editor: Hemant V. Ghate

Manuscript details:

Ms \# 02188

Received 26 April 2009

Final received 15 October 2010

Finally accepted 23 October 2010

Citation: Vyjayandi, M.C., R.S. Rajeesh, P. Sajin John \& M.M. Dhanasree (2010). On a collection of praying mantids (Insecta: Mantodea) from Goa, India, with new distribution records. Journal of Threatened Taxa 2(12) $1325-1329$

Copyright: (c) M.C. Vyjayandi, R.S. Rajeesh, P. Sajin John \& M.M Dhanasree 2010. Creative Commons Attribution 3.0 Unported License JoTT allows unrestricted use of this article in any medium for non-profit purposes, reproduction and distribution by providing adequate credit to the authors and the source of publication.

Acknowledgements: We gratefully acknowledge the Department of Science and Technology, Govt. of India for providing research grant. We sincerely thank the authorities of Providence Women's College, Kozhikode, Kerala, India, for providing laboratory facilities.

\section{OPEN ACGESS | FREE DOWNLOAD (C) (i) (4)}

with daytime temperatures touching $35^{\circ} \mathrm{C}$. The monsoon rains arrive around early June and have a short cool season which lasts between mid-December and February.

Materials and Methods: The field survey to collect mantid specimens was carried out in the Salim Ali Bird Sanctuary, Bondla Wildlife Sanctuary (WS), Bhagavan Mahaveer WS and Cotigao WS. Goa, during the months of September and October 2008. The specimens were hand picked and collected by light traping. Detailed studies of the specimens were carried out with a Leica EZ4D Stereo Zoom Microscope and digital images were prepared using Olympus SP-350 8MP camera. The specimens were identified following Giglio-Tos (1927), Mukherjee et al. (1995), Henry (1932) and Ehrmann (2002). All the specimens studied are kept in the Zoology Department of Providence Women's College, Kozhikode, Kerala, India. Measurements are given in millimeter.

Results: The systematic list of the mantid species collected from Goa is as follows.

\section{Order: Mantodea}

Family: Hymenopodidae

Sub family: Acromantinae

\section{Acromantis insularis Giglio-Tos (Images 1, 2, 3 \& 4)}

Acromantis insularis Giglio-Tos, 1927. Das Tierreich.50: 526.

Specimens Examined: 2 females \& 1 male, 01.x.2008, Cotigao WS, Goa, India, coll. Vyjayandi \& party.

Diagnostic characters: Generally greenish-brown, head triangular, $2 x$ wider than high, eyes rounded bulging, metazona $3 x$ longer than prozona, edge of the pronotum with fine tubercles; disc smooth; fore coxa as long as

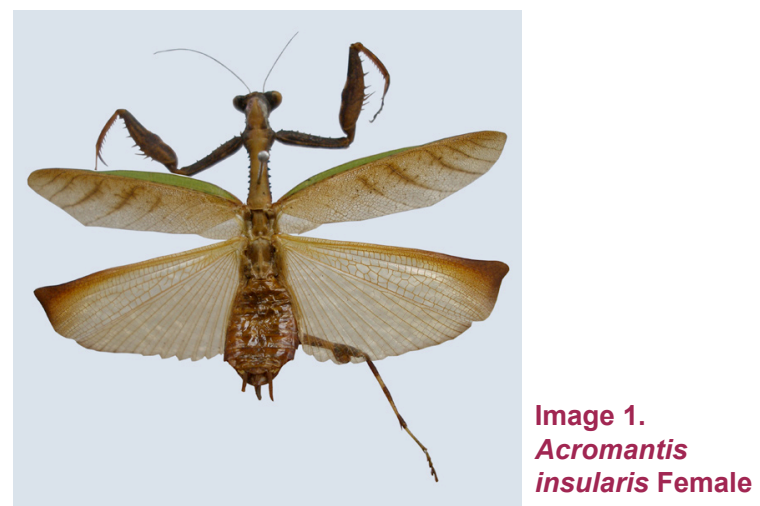

Abbreviations: FW - Fore wing; PN - Pronotum; TL - Total Length; WS - Wildlife Sanctuary. 


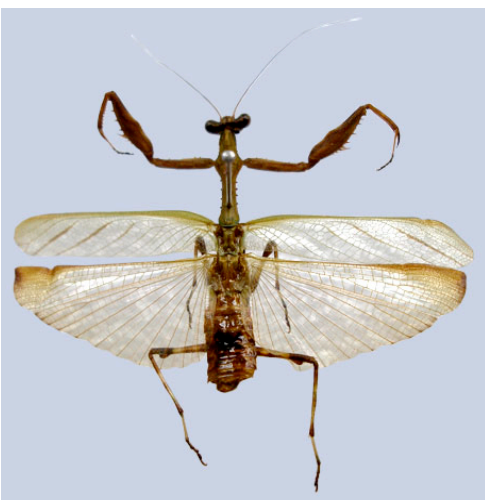

Image 2. Acromantis insularis Male

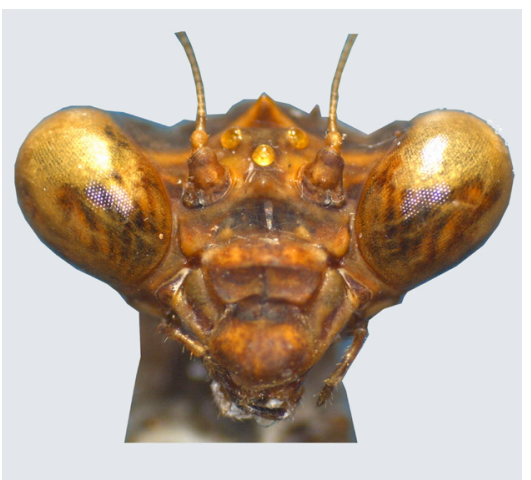

Image 3. Acromantis insularis Head - frontal view

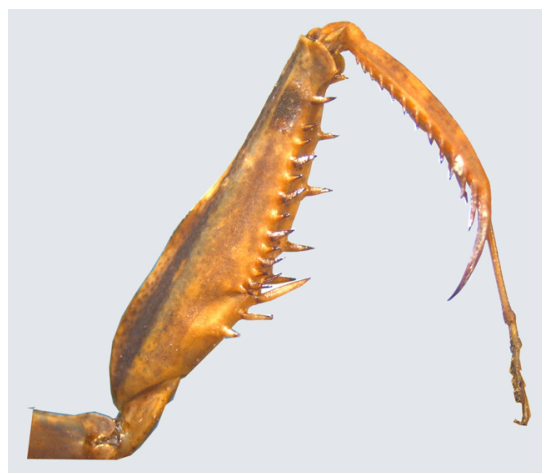

Image 4. Acromantis insularis Fore - femur metazona, 4-5 spines present in the anterior margin. Femur: dorsal middle region with a hump, four external spines, four discoidal spines and 13 internal spines, longer internal spines black at tip and base, claw groove placed at proximal end, wings longer than abdomen; fore wings less densely reticulated, costal area truncated at tip.

Measurements: Male: TL - 26mm, PN - 9mm, FW $21 \mathrm{~mm}$; Female: TL - 32mm, PN - 12mm, FW - 26mm.

Distribution: India (Goa (current study), Tamil Nadu, Kerala, Karnataka), Indonesia (Java, Sumatra).

\section{Family: Mantidae}

Sub family: Amelinae

\section{Amantis saussurei (Bolivar) (Images 5, 6 \& 7)}

Amantis saussurei: Giglio-Tos, 1927. Das Tierreich. 50: 171.

Gonypeta saussurei: Kirby, 1904. Cat. Orth. Brit. Mus., 1: 224

Specimens examined: 2 females, Bagavan Mahaveer WS, Mollem, Goa, India, 28.ix.2008, coll. Vyjayandi \& party.
Diagnostic characters: Dark brown; head eyes round, lateral lobes prominent; pronotum elliptical with a dorsomedian dark brown band, two tubercles at base of metazona dorso-medially, fore leg entirely setaceous, femur triangular, anterior margin almost straight with 11 internal, four external, four discoidal spines; tibia with 11 internal and nine external spines; both wings highly reduced.

Measurements: female: TL - 17mm, PN - 4mm.

Distribution: India (Goa (current study), Kerala, Andhra Pradesh, and Tamil Nadu).

Remarks: Mostly observed and collected during night.

\section{Elmantis trincomaliae (Saussure) (Images 8, 9, 10 \& 11)}

Elmantis trincomaliae: Beier, 1956. Verh. Naturf. Ges. Basel, 67(1): 35.

Elmantis trincomaliae: Giglio-Tos, 1927. Das Tierreich, 50: 178.

Elmantis trincomaliae: Giglio-Tos, 1915. Bull. Soc. Entomol. Ital., 46: 161.

Specimens examined: 3 males \& 2 females, 26.ix.2008,

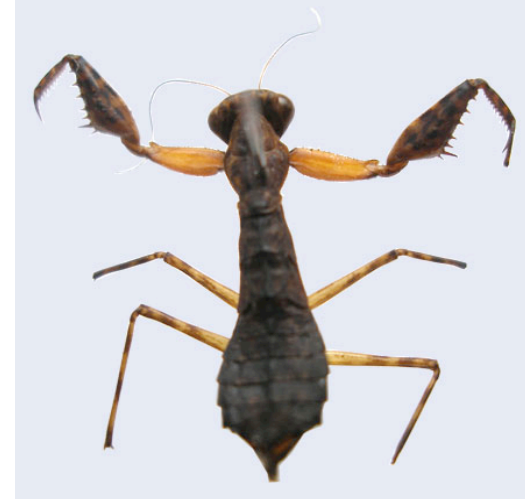

Image 5. Amantis saussurei Female entire view)

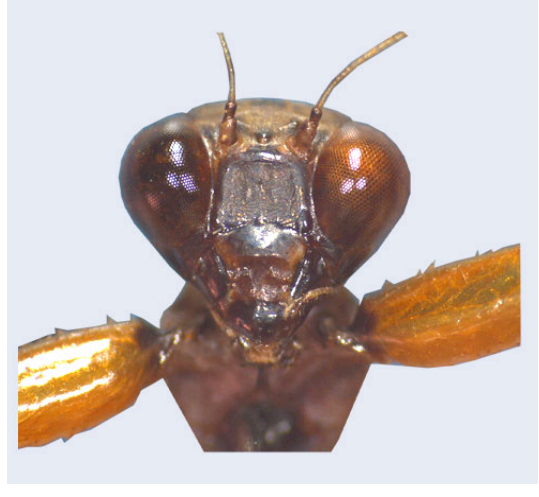

Image 6. Amantis saussurei Head frontal view

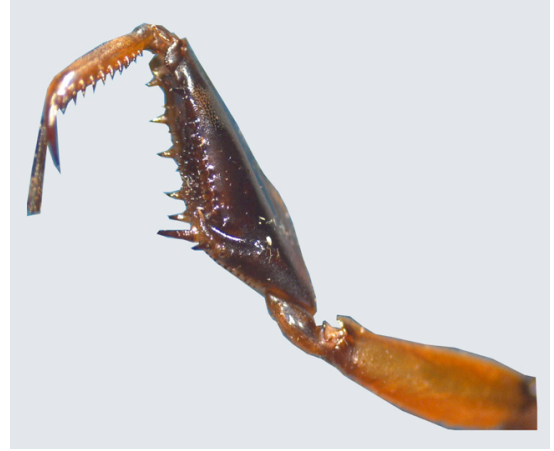

Image 7. Amantis saussurei Foreleg 


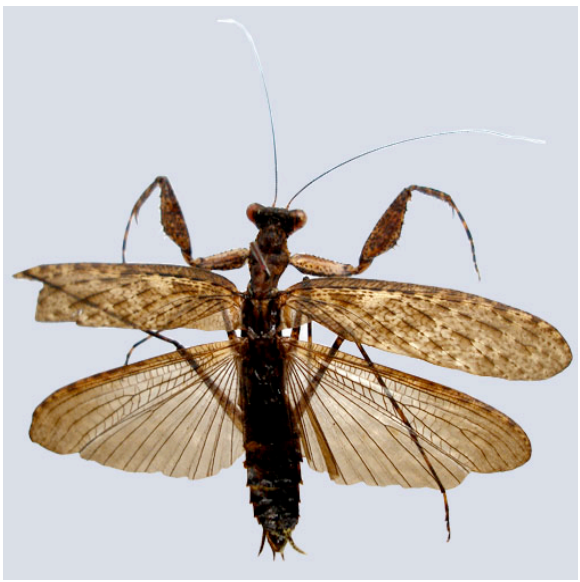

Image 8. Elmantis trincomaliae Male

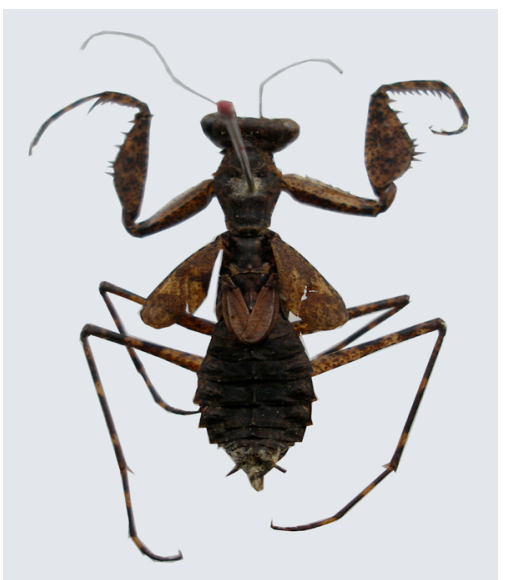

Image 9. Elmantis trincomaliae Female

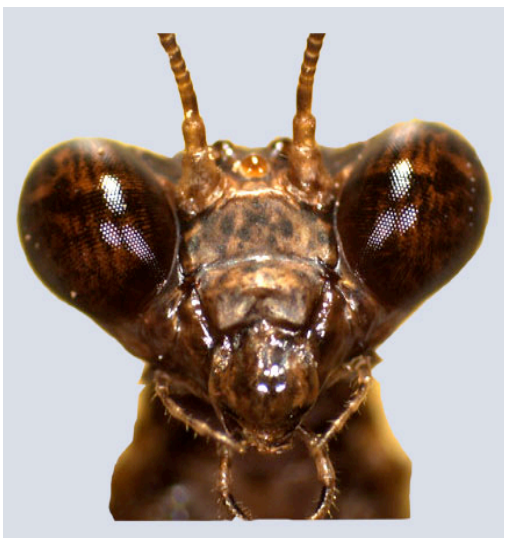

Image 10. Elmantis trincomaliae Head - frontal view

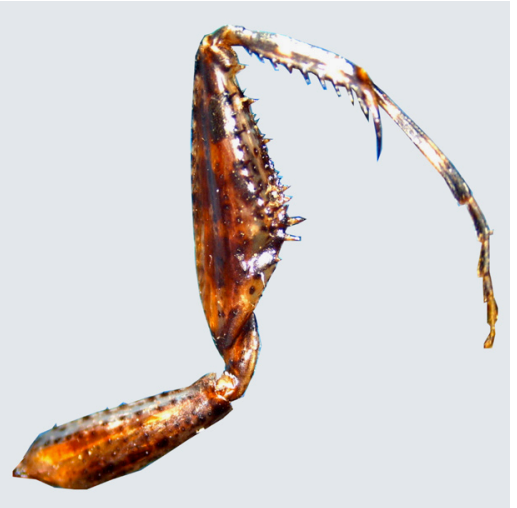

Image 11. Elmantis trincomaliae foreleg

Bondla WS, Goa, India, coll. Vyjayandi \& party; 2 males \& 2 females, 28.ix.2008, 29.ix.2008, Bagavan Mahaveer WS, Mollem, Goa, India, coll. Vyjayandi \& party; 1 female, 01.x.2008, Cotigao WS, Goa, India, coll. Vyjayandi \& party.

Diagnostic characters: Generally brown with dark brown dots and patches, head 1.6x wider than high; vertex smooth, lateral lobes distinct; eyes rounded,pronotum medium sized, slightly longer than fore coxa, fore coxa with 8-9 small spines, carinated; trochanter small; fore femur triangular, fore femora with four external spines (first two closely placed), four discoidal spines (first discoidal spine shorter than second), 12 external spines, claw groove placed in the proximal half. Wings hyaline, longer than abdomen. Females are brachypterous.

Measurements: Male - TL- 25mm, PN - 5mm, FW 22mm, Female - TL - 27mm, PN - 7mm, FW - 6mm.

Distribution: India (Goa (current study), Andhra Pradesh, Karnataka, Kerala, Maharashtra, Tamil Nadu), Sri Lanka (Trincomalee).

Remarks: Female specimens are little darker than males and short-winged.
Family: Liturgusidae Sub family: Liturgusinae

\section{Humbertiella similis Giglio-Tos} (Fig: 12, 13, 14 \& 15)

Humbertiella similis: Giglio-Tos, 1927. Das Tierreich, 50: 65 .

Humbertiella similis: Giglio-Tos, 1917. Bull. Soc. Entomol. Itl., 48: 83.

Diagnostic characters: Body generally bark brown dorsally, pale brown ventrally, vertex smooth, four grooved, lateral lobe slightly elevated from level of vertex forms obtuse tubercle, eyes large, pronotum broad, a little longer than fore coxa, dentate laterally, metazona about 2x longer than prozona, disc with 6-8 indistinct tubercles, fore coxa short, a little swollen ventrally, lower margin denticulate, internal apical lobe divergent; femur stout swollen proximally, with 14 ( 7 short, 7 large) internal, four external, four discoidal spines, margin between external spines dentate; claw groove proximally placed; tibia slender with 10 internal, nine external spines, wings hyaline, longer than abdomen.

Measurements: Male: TL - 30mm, PN - 5.5mm, FW 27mm; Female: TL - 34mm, PN - 6.5mm, FW - 22mm,

Specimens examined: 1 male and 1 female, 30.ix.2008, ICAR, Old Goa, India, coll. Vyjayandi \& party.

Distribution: India (Goa (current study), Himachal Pradesh, Jammu, Kerala, Madhya Pradesh, Orissa, Uttar Pradesh), Nepal, Sri Lanka.

Remarks: The body size of both male and female almost same. Both wings of females are little shorter than abdomen, fore wing opaque with irregular costal veinlets; hind wing sub opaque. 


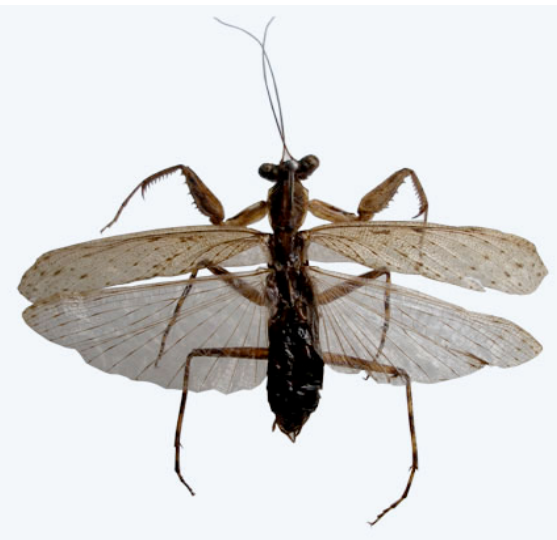

Image 12. Humbertiella similis Male

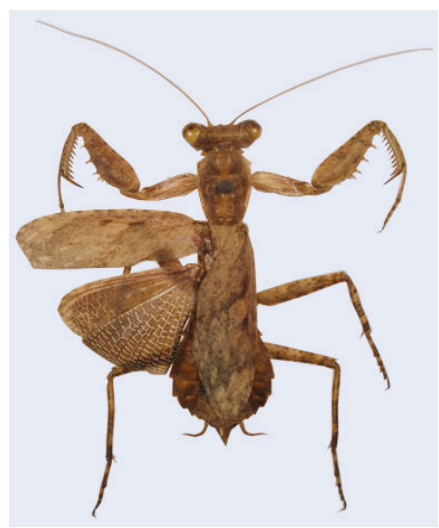

Image 13. Humbertiella similis Female

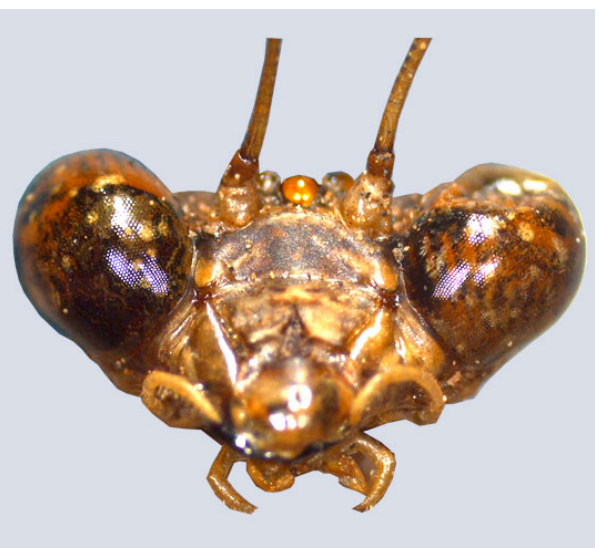

Image 14. Humbertiella similis Frontal view

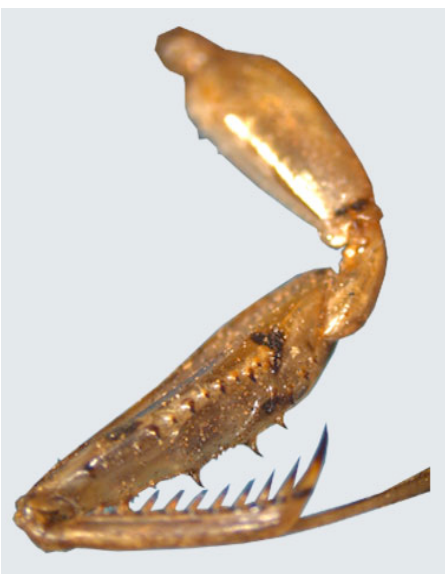

Image 15. Humbertiella similis Fore-leg

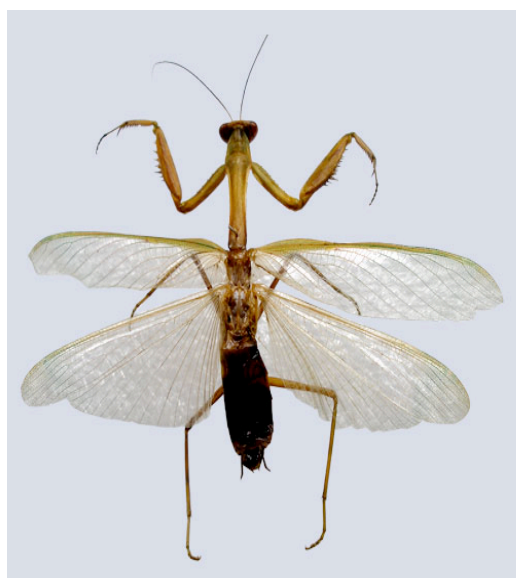

Image 16. Hierodula ventralis Male

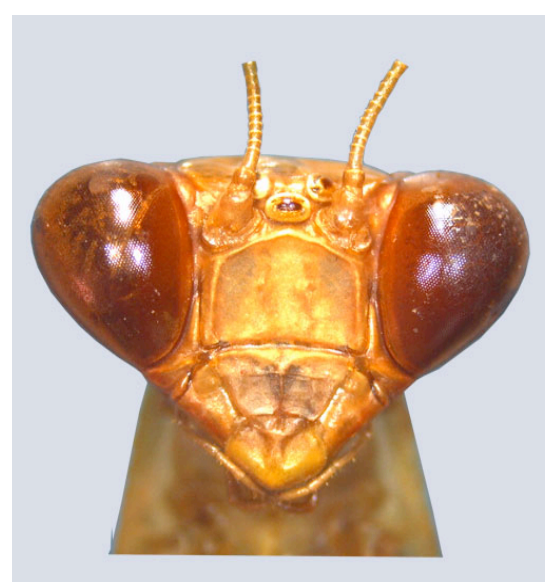

Image 17. Hierodula ventralis Frontal view
Family: Mantidae

Sub family: Mantinae

\section{Hierodula ventralis Giglio Tos (Fig: 16, 17, 18 \& 19)}

Hierodula ventralis Giglio Tos, 1912. Bull. Soc. Entomol.Ital.,43: 82.

Hierodula ventralis Giglio Tos, 1927. Das Tierreich, 50: 443.

Hierodula ventralis Werner, 1935. Proc. Zool. Soc. Lodon, 1935: 495-498.

Diagnostic characters: Generally light green, head wider than high, vertex smooth, lateral lobes distinct; eyes globular, large, bulging; pronotum long; metazona 4.2x longer than prozona; fore coxa with six spinules; fore femur with four external spines, four discoidal spines and 15 internal spines, last two smaller spines closely placed, wings longer than the abdomen; fore wing semi hyaline; hind wing transparent. $44 \mathrm{~mm}$.
Specimens examined: 1 male, 26.ix.2008, Bondla WS, Goa, India, 30m, coll. Vyjayandi \& party.

Distribution: Goa (current study), Chandigarh, Kerala, Madhya Pradesh, Maharashtra.

\section{Family: Toxoderidae}

Sub family: Toxoderinae

\section{Cheddikulama straminea Henry}

(Fig: 20, 21 \& 22)

Cheddikulama straminea Henry, 1932. Spolia Zeylanica, 17(1):14-18, pl.II,f.2; pl.V,f.2

Diagnostic characters: Generally straw colored, appearing as dry grass; head large, somewhat flattened, broader than high, eye mammiform, pronotum long, slender with dorsomedian carina; lateral margin dentate; metazona a little longer than fore coxa, upper and lower margin of coxa dentate, internal apical lobes divergent, coxa finely granulated with 13 internal (6 short, 7 large), four discoidal, first two closely placed, four external spines, margin between external spines serrated; claw 


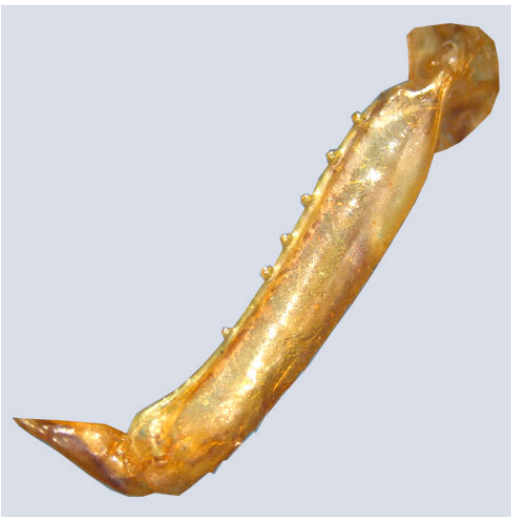

Image 18. Hierodula ventralis Fore coxa

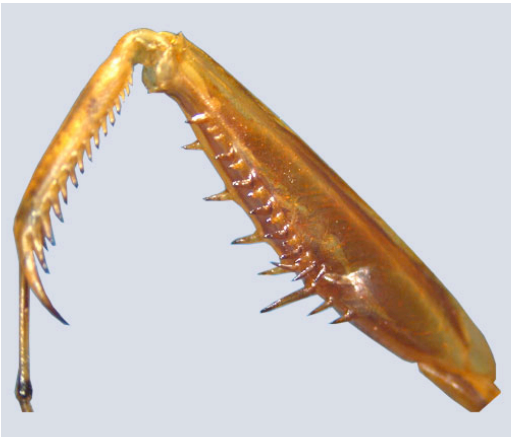

Image 19. Hierodula ventralis Fore leg

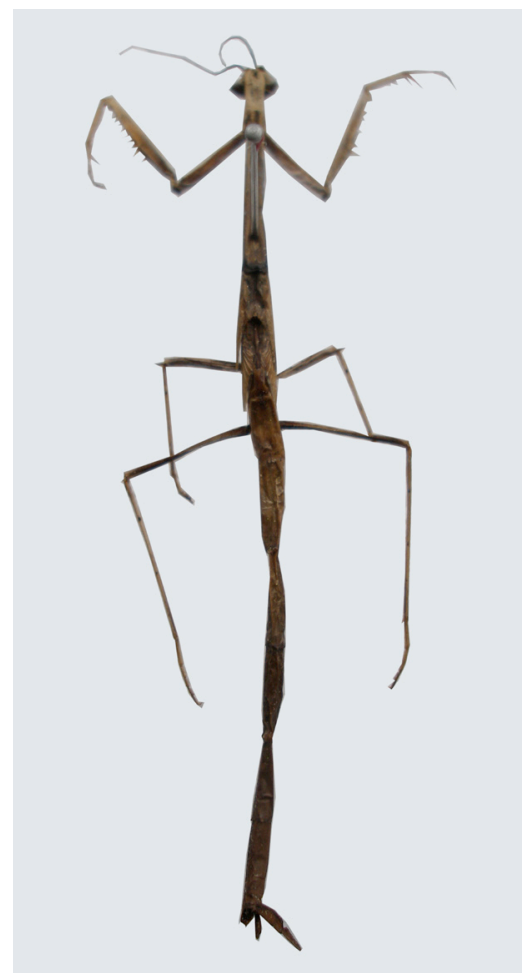

Image 20. Cheddikulama straminea Frontal view

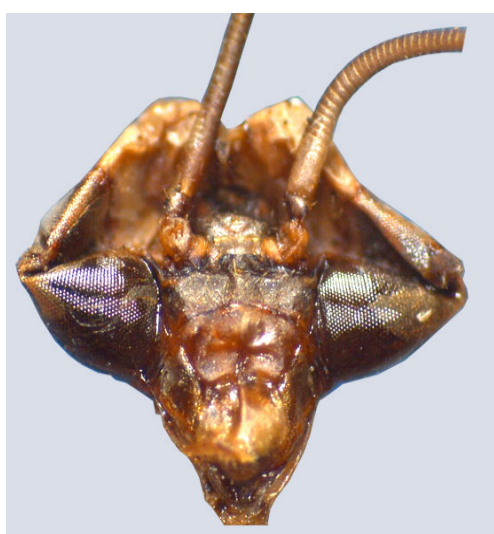

Image 21. Cheddikulama straminea Frontal view

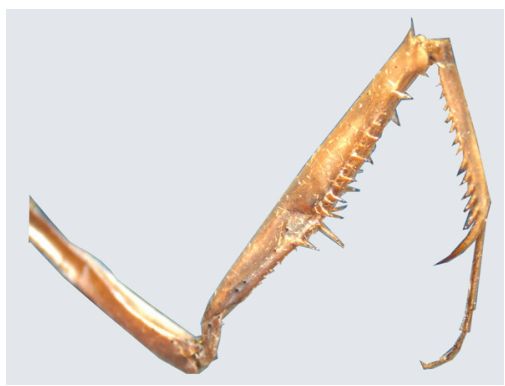

Image 22. Cheddikulama straminea Fore femur groove proximally placed, tibia with 16 internal, 11 external spines; both wing shorter than abdomen; fore wing opaque at costal area, anal, discoidal area semi transparent; hind wing hyaline with characteristic colorations.

Specimens examined: Male, 26.ix.2008, Bondla WS, Goa, India, 30m, coll. Vyjayandi \& party.

Distribution: India: (Goa (current study), Kerala, Uttar Pradesh), Sri Lanka (Mannar, Cheddikulum).

\section{REFERENCES}

Beier (1956). Verhandelingen over de natuurlijke Geschieden der Nederlandsche overzeeshe Bezittingen, door de Leden der natuurundig Commissie in Oost Indie en andere Schrijvers. Zoologie 67(1): 35.

Ehrmann, R. (2002). Mantodea: Gottesanbeterinnen der Welt. Natur und Tier - Verlag GmbH An der Kleimannbrücke, Münster, Berlin, 519pp.

Giglio-Tos, E. (1927). Mantidae, pp. 171-526. In: Das Tierreich. Mantidae. Walter de Gruyter \& Co.

Henry, G.M. (1932). Observations on some Ceylonese Mantidae with descriptions of new species. Spolia Zeylanica 17: 1-18.

Kirby, W.F. (1904). A Synonymic Catalogue of Orthoptera Volume 1. British Museum, London, 224pp.

Mukherjee, T.K, A.K. Hazra \& A.K. Ghosh (1995). The mantid fauna of India (Insecta: Mantodea). Oriental Insects 29: 208320.

Werner, F. (1935). Farther communication on Indian Mantids or Praying Insects. Proceedings of the Zoological Society of London 105: 495-498. 